\title{
Medial temporal lobe epilepsy associated with cortical dysplasia extending from the medial temporal lobe to the fornix: a case report
}

\author{
Takato Morioka ${ }^{1}$, Nobutaka Mukae ${ }^{1}$, Tetsuro Sayama ${ }^{1}$, Takeshi Hamamura ${ }^{1}$, Asako Nomoto ${ }^{2}$, \\ Kuniko Yamamoto ${ }^{3}$, Tomomi Kido ${ }^{3}$, Tatsumi Yamaguchi ${ }^{4}$, Satoshi O Suzuki ${ }^{5}$, Tomio Sasaki ${ }^{6}$ \\ ${ }^{1}$ Department of Neurosurgery, Kyushu Rosai Hospital, Kitakyushu, Japan \\ ${ }^{2}$ Department of Radiology, Kyushu Rosai Hospital, Kitakyushu, Japan \\ ${ }^{3}$ Department of Clinical Laboratory, Kyushu Rosai Hospital, Kitakyushu, Japan \\ ${ }^{4}$ Yamaguchi Neurosurgical Clinic, Kitakyushu, Japan \\ ${ }^{5}$ Department of Neuropathology, Graduate School of Medical Sciences, Kyushu University, Fukuoka, Japan \\ ${ }^{6}$ Department of Neurosurgery, Graduate School of Medical Sciences, Kyushu University, Fukuoka, Japan
}

Key words: medial temporal lobe epilepsy, cortical dysplasia, hippocampus, fornix

Received Jan. 18, 2010; Accepted March 1st, 2010 ; Published online March 29, 2010

\section{Summary}

We report a case of lesional medial temporal lobe epilepsy (TLE) associated with cortical dysplasia extending from the right medial temporal lobe to the fornix via the basal and medial aspects of the frontal lobe and septum pellucidum. A 23-year-old man had had medically intractable psychomotor seizures since 11 years of age. Interictal EEG demonstrated paroxysmal activities in the right temporal region, and functional imaging by positron emission tomography with $\left[{ }^{18} \mathrm{~F}\right]$ fluorodeoxyglucose revealed a functional deficit zone in the apex and medial part of the right temporal lobe. An intraoperative electrocorticogram demonstrated frequent paroxysmal activities in the medial temporal lobe. An anterior temporal lobectomy was performed, with an additional hippocampectomy including the focus of the

Corresponding author: Takato Morioka, M.D., Ph.D.

Department of Neurosurgery, Kyushu Rosai Hospital, 1-3-1 Kuzuharatakamatsu, Kokuraminami-ku, Kitakyushu-city, Fukuoka 800-0296, Japan. Tel: +81-93-471-1121; Fax: +81-93-473-0627; E-mail: takato@med.kyushu-u.ac.jp 
paroxysmal activities on the intraoperative hippocampal EEG. Postoperatively, he became seizure-free. The surgical strategies for intractable TLE with widely distributed cortical dysplasia lesion are discussed.

\section{Introduction}

Cortical dysplasia (CD), especially focal cortical dysplasia (FCD), is a congenital malformation of cortical development that is a frequent cause of refractory epilepsy in both children and adults. With advances in structural and functional neuroimaging techniques, such lesions are increasingly being identified as the causes of intractable epilepsy in patients undergoing epilepsy surgery [1-4]. Since Taylor et al. in 1971 [5] first described the pathological characteristics of FCD after reviewing resected specimens from patients suffering from intractable temporal lobe epilepsy (TLE), the temporal lobe has been considered to be one of the most common sites of FCD [1]. We report a case of lesional TLE associated with a continuous CD lesion extending from the right medial temporal lobe to the fornix via the basal and medial aspects of the frontal lobe and septum pellucidum. The surgical strategies for intractable TLE with a widely distributed CD lesion are discussed.

\section{Case report}

A 23-year-old right-handed man had had psychomotor seizures since 11 years of age. Although his seizures were almost completely controlled with valproate monotherapy, they recurred at 20 years of age. His seizures began with a headache, followed by cloudy con- sciousness, hand motor automatism and abnormal behaviors such as walking around in the room. Secondary generalized tonic-clonic seizures were rarely seen. He was treated with various drugs including valproate, carbamazepine, zonisamide and clobazam, but his seizures became medically intractable. While receiving optimal dosages of zonisamide and clobazam, he had weekly psychomotor seizures.

A presurgical evaluation was performed. Neurological findings were normal except a mild disturbance of verbal memory function (verbal memory score 79 and visual memory score 114 on the WMS-R). His total IQ was 82 (PIQ 94 and VIQ 75). Interictal EEG revealed sporadic sharp waves of small amplitude in the right temporal legion. Ictal event was not captured. Although a CT scan failed to reveal any abnormality, MR images with fluidattenuated inversion recovery sequence (FLAIR) demonstrated a continuous hyperintense area extending from the right medial temporal lobe to the fornix via the basal and medial aspects of the frontal lobe and septum pellucidum (Fig. 1A, B). The lesion in the medial temporal lobe was mainly located in the amygdala, uncus and temporal stem, while the hippocampus was spared. Positron emission tomography with $\left[{ }^{18} \mathrm{~F}\right]$ fluorodeoxyglucose (FDG-PET) [6] depicted hypometabolism in the apex and medial part of the right temporal lobe (Fig. 1C). Single photon emission computed tomography with $\left[{ }^{123} \mathrm{I}\right]$ iomazenil (IMZ-SPECT) [7] demonstrated low uptake in the apex of the right temporal lobe (Fig. 1D, E). Although ictal EEG could not be 

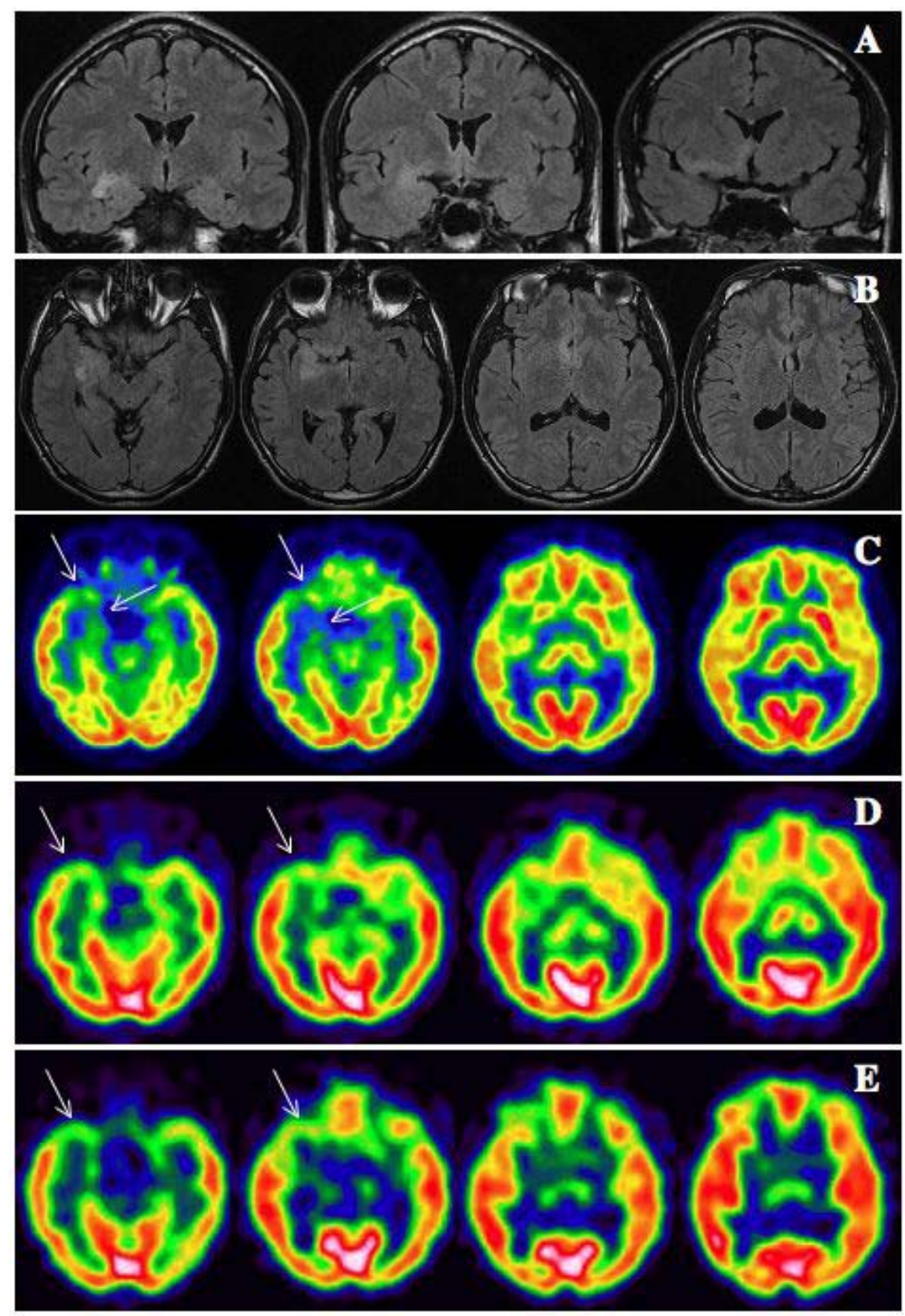

Figure 1.

A, B: Coronal [A] and oblique (along with the long axis of the hippocampus) [B] views of MR images with fluid-attenuated inversion recovery sequence (FLAIR). The FLAIR-MR images demonstrate a continuous hyperintense area extending from the right medial temporal lobe to the fornix via the basal and medial aspects of the frontal lobe and septum pellucidum.

C: $\quad$ Positron emission tomography with $\left[{ }^{18} \mathrm{~F}\right]$ fluorodeoxyglucose (FDG-PET) at a comparable level to that in [B]. FDG-PET depicts hypometabolism in the apex and medial part of the right temporal lobe (white arrows).

D, E: Single photon emission computed tomography with $\left[{ }^{123} \mathrm{I}\right]$ iomazenil (IMZ-SPECT) at a comparable level to [B] at 20 minutes [D] and 3 hours [E] after injection of IMZ. Both IMZ-SPECT images demonstrate low uptake in the apex of the right temporal lobe (white arrows). 
recorded, the findings of the interictal EEG and functional imaging such as FDG-PET and IMZ-SPECT were consistent with those of right lesional TLE. Thus, epilepsy surgery for right temporal lobe was scheduled.

At 23 years of age, through a right frontotemporo-parietal craniotomy, an intraoperative electrocorticogram (ECoG) was recorded from the medial, basal and lateral aspects of the temporal lobe, and demonstrated frequent paroxysmal activities over the medial temporal lobe (Fig. 2). Resection of the middle and inferior temporal gyri was performed at 3.5 $\mathrm{cm}$ from the temporal apex, and the inferior horn of the lateral ventricle was exposed (Fig. 3A). The FLAIR hyperintense lesion in the medial temporal lobe, which was confirmed using a neuronavigation system, was resected for pathological diagnosis. An EEG recorded from the hippocampus [8] showed frequent paroxysmal activities (Fig. 3); therefore an additional hippocampectomy was performed. Since the focus of the paroxysmal activities was located at $2 \mathrm{~cm}$ from the hippocampal tip, the extent of the hippocampal resection was $2.5 \mathrm{~cm}$ from the hippocampal tip including the

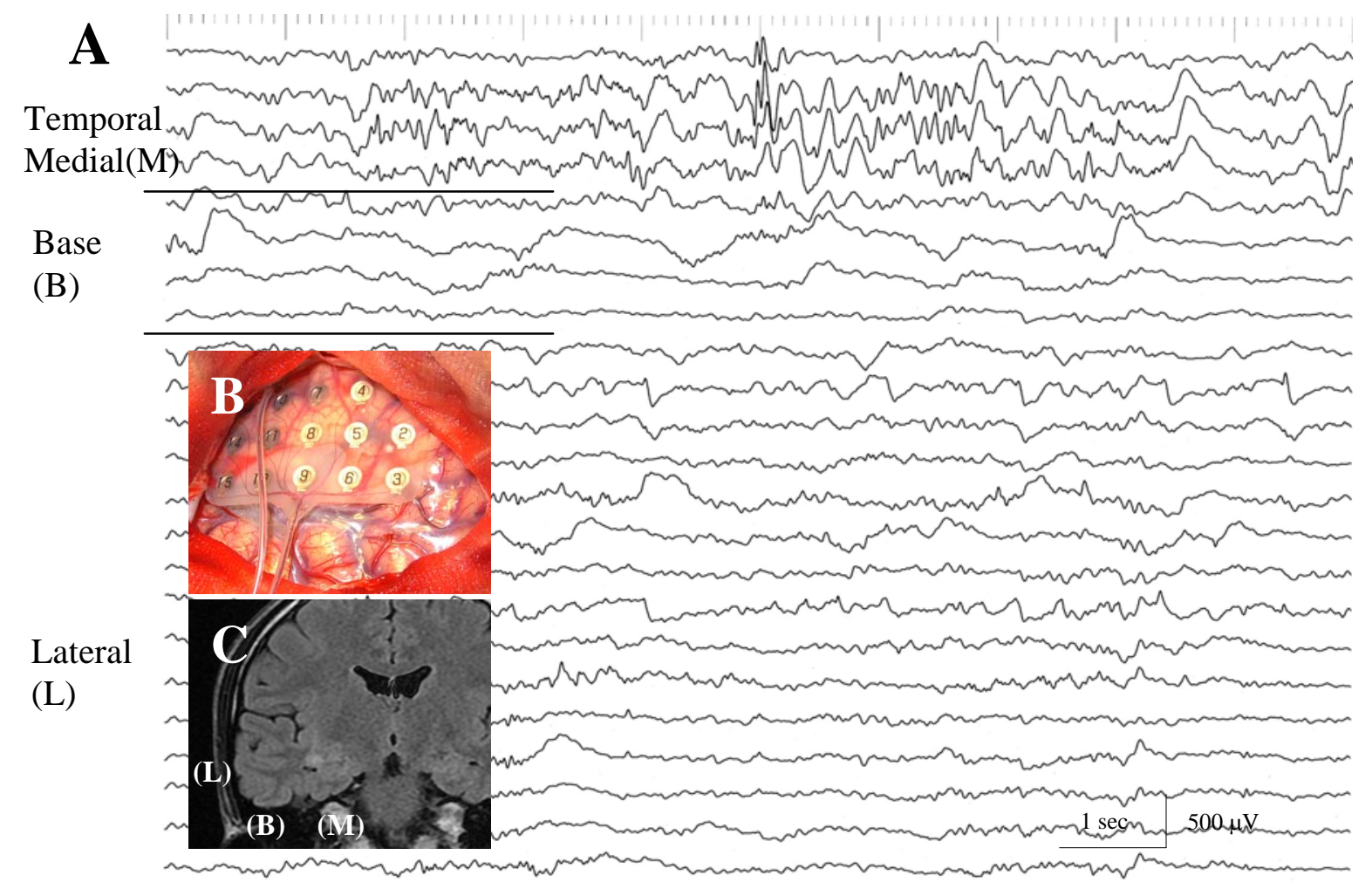

Figure 2.

A: Intraoperative electrocorticography (ECoG) with nasion reference. Recording electrodes are placed on the medial (M), basal (B) and lateral (L) aspects of the temporal lobe. Frequent paroxysmal discharges are observed in the medial temporal lobe.

B: An intraoperative photograph shows the grid electrode placed on the lateral temporal lobe.

C: The recording sites are indicted in a coronal section of the FLAIR images. 

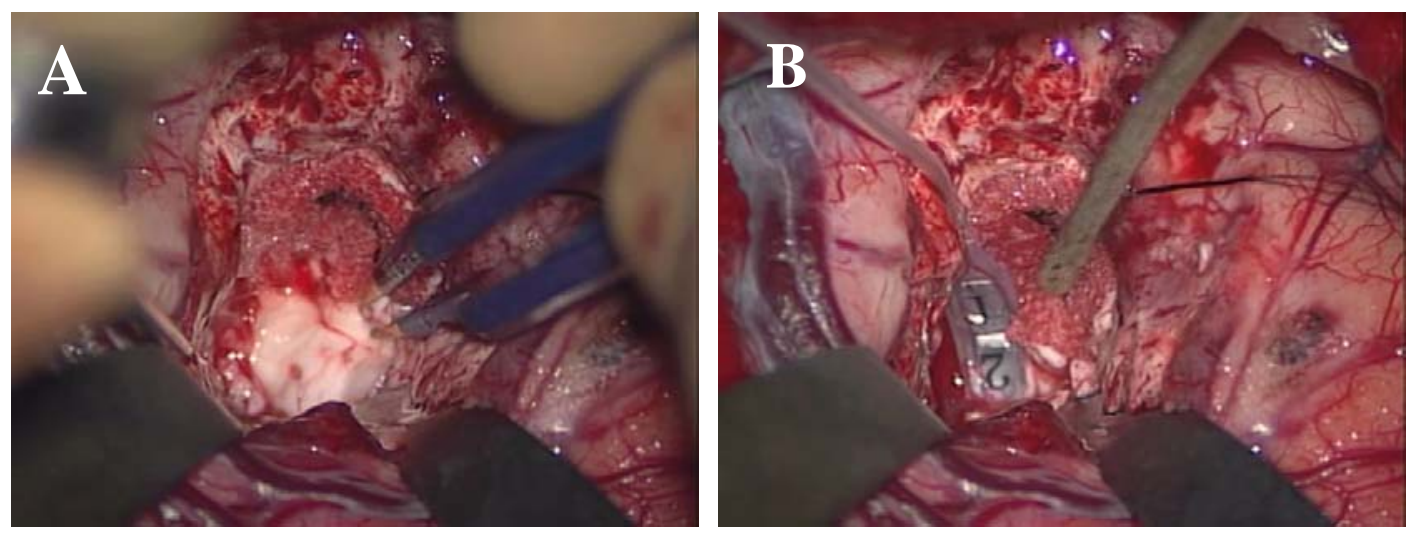

C

$0.5 \mathrm{~cm}$

1.0

1.5

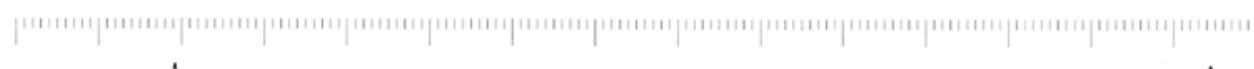

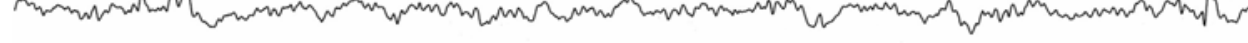

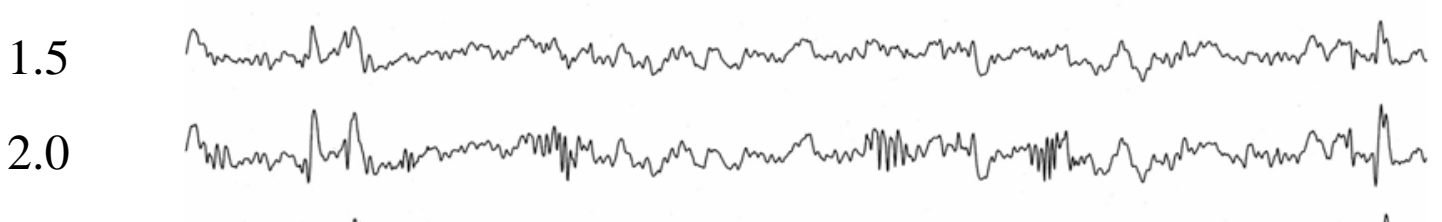

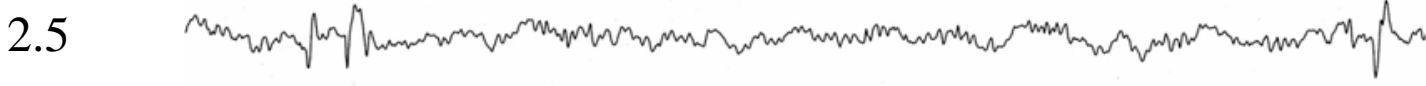

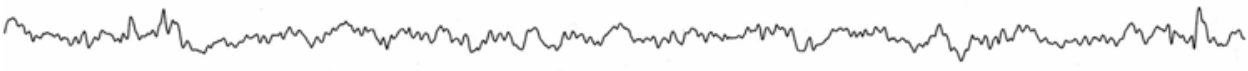

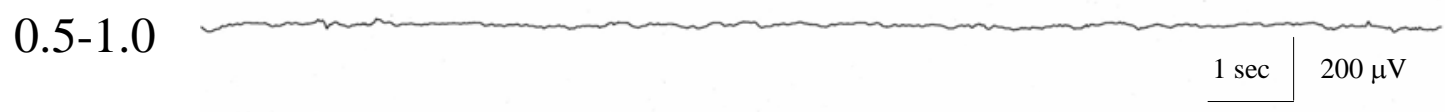

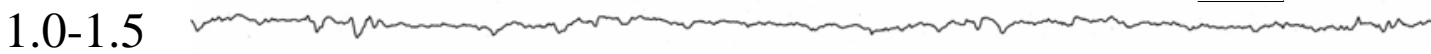

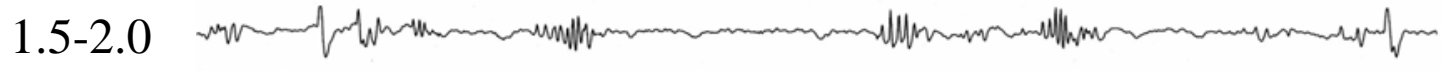

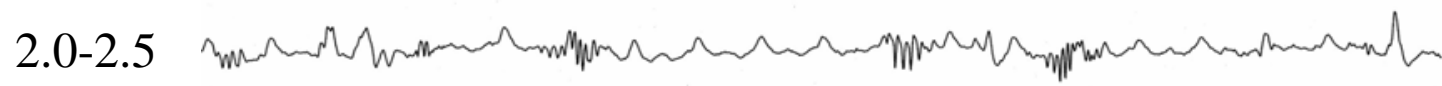

Figure 3.

A: An intraoperative photograph showing the exposed hippocampus after resection of the lateral temporal lobe.

B: A strip electrode is placed on the hippocampus.

C: An intraoperative electrocorticogram of the hippocampus. The numbers indicate the distances of the recording electrodes from the hippocampal tip. The upper five tracings are monopolar recordings with nasion reference and the lower three tracings are bipolar recordings. There are frequent paroxysmal discharges, with the highest voltage in the monopolar recording and phase reversal in the bipolar recording at $2.0 \mathrm{~cm}$ posterior to the hippocampal tip. 
paroxysmal activity focus. ECoG recording from the remaining lesions such as frontal base was not attempted because of technical difficulty.

Postoperatively, the patient had transient depressive state and zonisamide was discontinued. With monotherpy of clobazam, he was seizure-free during 6 months after surgery. His cognitive function was unchanged compared to the preoperative state (verbal memory score 81 and visual memory score 110 on the WMS-R, total IQ 85, PIQ 96 and VIQ 78).
Histopathological examination of the FLAIR hyperintense lesion in the medial temporal cortex showed dysmorphic neurons with clumped Nissl bodies (Fig. 4A), occasional clustering of the neurons (Fig. 4B) and increased neuronal satellitosis (Fig. 4A, C). Since no balloon cells were present, the findings indicated CD of Palmini type IIA [9]. Neither hippocampal sclerosis nor microdysgenesis was seen in the resected hippocampus (Fig. 4D).
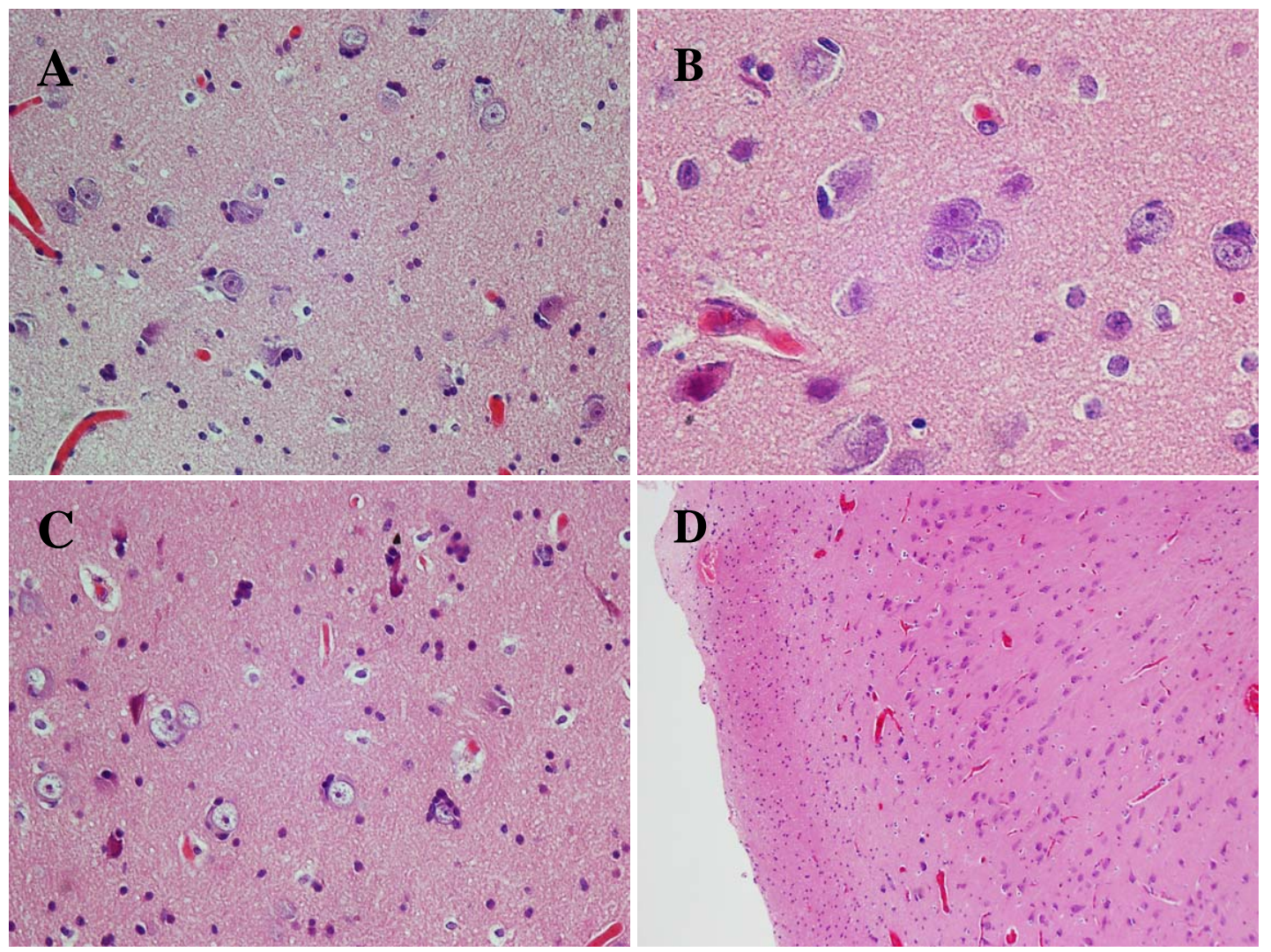

Figure 4.

A-C: Histopathological findings of the FLAIR hyperintense lesion in the medial temporal cortex.

A: Dysmorphic neurons showing clumped Nissl bodies are observed (original magnification 40×, H\&E staining).

B: Clustering of the neurons is occasionally seen (original magnification $80 \times, \mathrm{H} \& \mathrm{E}$ staining).

C: Increased neuronal satellitosis is noted (original magnification 40×, H\&E staining).

D: Coronal section of the CA1 region of the hippocampus.

Neither neuronal loss nor microdysgenesis is seen (original magnification 10×, H\&E staining). 


\section{Discussion}

The typical features of type II FCD on MR images include focal cortical thickening, blurring of the gray-white matter junction and T2-weighted hyperintensity of the subcortical white matter that often tapers toward the ventricle $[10,11]$. In the present case, the FLAIR hyperintense lesion extended from the right medial temporal lobe to the fornix via the basal and medial aspects of the frontal lobe and septum pellucidum, and lacked the typical MR findings of type II FCD. We found no previous report describing this kind of continuous distribution of a CD lesion. Recently, transneuronal degeneration of the limbic system, such as atrophy of the fornix via the circuit of Papez, has been clearly demonstrated in TLE patients with hippocampal sclerosis by MR imaging [12, 13]. In the present case, transneuronal degeneration could be ruled out, since the circuit of Papez was spared except for the fornix.

In the present case, the aim of the surgery was management of the intractable epilepsy as well as histological confirmation of the continuous lesion. The seizure semiology and findings of the interictal EEG and functional imaging such as FDG-PET and IMZ-SPECT $[6,7]$ were consistent with those of TLE, although the MR lesions in the temporal lobe extended outside of the temporal lobe. In this situation, invasive video-intracranial EEG monitoring with subdural grids or depth electrodes may be indicated to aid localization of the epileptogenic zone [1-3, 14]. However, placement of the intracranial electrodes in the deep-seated continuous lesion such as fornix was too invasive, and surgery for the TLE under the guidance of intraoperative ECoG [8] was selected.

Since the findings of the intraoperative ECoG were typical findings for TLE, the FLAIR hyperintense lesion of the medial temporal cortex was resected through an anterior temporal lobectomy. Another issue is the necessity of an additional hippocampectomy [8]. On MR images, the FLAIR hyperintense lesion in the medial temporal lobe was located adjacent to the hippocampus, but the hippocampus was spared. Since the intraoperative hippocampal EEG showed frequent paroxysmal activities, an additional hippocampectomy including the paroxysmal activity focus was performed. The rationale for hippocampectomy based on intraoperative hippocampal EEG remains in dispute [8]. Usui et al. [15] reported that hippocampal interictal (not intraoperative) epileptiform discharges during chronic intracranial EEG recording are universal findings in patients with lesional lateral TLE. However, the potential risks and benefits of the additional hippocampectomy must be considered carefully on a case-by-case basis, and hippocampal resection may be indicated with the guidance of presurgical multimodal examinations as well as intraoperative hippocampal EEG [8].

In this case, neither hippocampal sclerosis nor microdysgenesis was noted histologically. In other words, "dual pathology" was not noted. Dual pathology does not merely indicate the morphological coexistence of hippocampal sclerosis with an extrahippocampal lesion, but also indicates the pathophysiologi- 
cal mechanisms such as the phenomena of kindling and secondary hippocampal epileptogenesis [14]. In the present case, we postulate that CD initially caused the repeated seizures and that the hippocampus gained secondary and independent epileptogenesis without morphological changes. For best clinical practice, the necessity of an additional hippocampectomy should be determined by integrated assessment of the clinical findings, MR and functional imaging, as well as interictal and ictal EEG findings, together with invasive or intraoperative ECoG findings [8, 14].

In our case, the total CD lesion, especially the lesion in the fornix, could not be resected. However, the epileptogenic hippocampus was resected through an anterior temporal lobectomy. The patient achieved good seizure control during the first 6 months after surgery. However, since the completeness of resection of a CD lesion appears to have the most predictive power for long-term seizure-free outcome [1-3], a long-term follow-up should be performed in the present case.

\section{Acknowledgments}

We thank Mr. Kohji Miyazoe, in the Department of Radiology, Kyushu Rosai Hospital for supporting our study.

\section{References}

[1] Diaz RJ, Sherman EMS, Hader WJ. Surgical treatment of intractable epilepsy associated with focal cortical dysplasia. Neurosurg Focus 2008; 25(3): E6.

[2] Duchowny M. Clinical, functional, and neurophysiologic assessment of dysplastic cortical networks: Implications for cortical functioning and surgical mamagement. Epilepsia 2009; 50(Suppl. 9): 19-27.

[3] Mathern GW. Challenges in the surgical treatment of epilepsy patients with cortical dysplasia. Epilepsia 2009; 50(Suppl. 9): 4550.

[4] Morioka T, Nishio S, Ishibashi H, Muraishi M, Hisada K, Shigeto H, Yamamoto T, Fukui M. Intrinsic epileptogenicity of focal cortical dysplasia as revealed by magnetoencephalography and electrocorticography. Epilepsy Res 1999 ;33: 177-187.

[5] Taylor DC, Falconer MA, Bruton CJ, Corsellis JA. Focal dysplasia of the cerebral cortex in epilepsy. J Neurol Neurosurg Psychiatry 1971; 34: 369-387.

[6] Hashiguchi K, Morioka T, Yoshida F, Kawamura T, Miyagi Y, Kuwabara Y, Sasaki, M, Koga H, Nagata S, Sasaki T. Thalamic hypometabolism on 18FDG-positron emission tomography in medial temporal lobe epilepsy. Neurol Res 2007; 29: 215222.

[7] Kaneko K, Sasaki M, Morioka T, Koga H, Abe K, Sawamoto H, Ohya N, Yoshiura T, Mihara F, Honda H. Pre-surgical identification of epileptogenic areas in temporal lobe epilepsy by 123I-iomazenil SPECT: A comparison with IMP-SPECT and FDGPET. Nucl Med Commun 2006; 27: 893899.

[8] Morioka T, Hashiguchi K, Nagata S, Miyagi Y, Yoshida F, Shono T, Mihara F, Koga H, Sasaki T. Additional hippocampectomy in the surgical management of intractable temporal lobe epilepsy associated with glioneuronal tumor. Neurol Res 2007; 29: 807-815.

[9] Palminia, Najm I, Avanzini G, Babb T, Guerrini R, Foldvary-Schaefer N, Jackson 
G, Luders HO, Prayson R, Spreafico R, Vinters HV. Terminology and classification of the cortical dysplasia. Neurology 2004; 62(Suppl 3): S2-8.

[10] Colombo N, Tassi L, Galli C, Citterio A, Lo Russo G, Scialfa G, Spreaflco R. Focal cortical dysplasias: MR imaging, histopathologic, and clinical correlations in surgically treated patients with epilepsy. AJNR Am J Neuroradiol 2003; 24; 724-733.

[11] Madan N, Grant PE. New directions in clinical imaging of cortical dysplasia. Epilepsia 2009; 50(Suppl. 9): 9-18.

[12] Kodama F, Ogawa T, Sugihara S, Kamba M, Kohaya N, Kondo S, Kinoshita T. Transneuronal degeneration in patients with temporal lobe epilepsy: evaluation by MR imaging. Eur Radiol 2003; 13: 2180-2185.

[13] Oikawa H, Sasaki M, Tamakawa Y, Kamei A. The circuit of Papez in mesial temporal sclerosis. Neuroradiology 2001; 43: 205210.

[14] Samura K, Morioka T, Hashiguchi K, Miyagi Y, Shigeto H, Sakata A, Tanaka S, Suzuki SO, Sasaki T. Temporal lobe epilepsy associated with'triple pathology’ of hippocampal sclerosis, focal cortical dysplasia and cavernoma in the ipsilateral frontal lobe. Epilepsy \& Seizure 2009; 2: 34-41.

[15] Usui N, Mihara T, Baba K, Matsuda K, Tottori T, Umeoka S, Nakamura F, Terada $\mathrm{K}$, Usui K, Inoue Y. Intracranial EEG findings in patients with lesional lateral temporal lobe epilepsy. Epilepsy Res 2008; 78: 82-91. 